\title{
ON THE SYMMETRY AXIOM FOR VALUES OF NONATOMIC GAMES
}

\author{
DOV MONDERER \\ Department of Mathematics \\ University of California, Los Angeles \\ Los Angeles, CA 90024, U.S.A \\ and \\ WILLIAM H. RUCKLE \\ Department of Mathematical Sciences \\ Clemson University \\ Clemson, SC 29634-1907, U.S.A. \\ (Received June 30, 1988)
}

ABSTRACT. In this paper, a weaker version of the Symmetry Axiom on BV, and values on subspaces of $\mathrm{BV}$ are discussed. Included are several theorems and examples.

KEY WORDS AND PHRASES. Aumann-Shapley value, set function and symmetry. 1980 AMS SUBJECT CLASSIFICATION CODE. 90013.

\section{INTRODUCTION AND STATEMENT OF RESULTS.}

It has been shown by Aumann and Shapley [1] that there is no value defined on the entire space BV. However, it was shown in Ruckle [2] that there do exist continuous, efficient projections from BV onto FA which satisfy a weaker form of the Symmetry Axiom. In this paper we shall pursue this phenomenon to a greater extent.

Throughout this paper we use the terminology and notation of Aumann and Shapley [1]. Let $(I, C)$ denote a standard measureable space which will remain fixed throughout the discussion. A symmetry of $(I, C)$ is a one to one bi-measureable transformation of $(I, C)$ onto itself. The group of all symmetries of (I,C) is denoted by G. For every $v$ in BV let $G(v)$ be the subgroup of all symmetries $\pi$ which preserve $v, 1 . e . v o \pi=v$. Let $Q$ be a symmetric linear subspace of $B V$, and let $\phi: Q \rightarrow$ FA be a value. By the Symmetry Axiom it follows that $G(v)$ is contained in $G(\phi v)$ for every $v$ in $B V$. This motivates us to define a measure group to be a group of symmetries $H$ for which there is $\mu$ in FA such that $H \subset G(\mu)$. A game $v$ will be called a valueable game if $G(v)$ is a measure group. A symmetric linear space of games is called a valueable space if each of its members is a valueable game.

The proof of the result of Aumann and Shapley cited in the first paragraph can be analyzed as follows: First it is shown that $G$ is not a measure group. Then the unanimity game $w$ is defined as the game for which $w(I)=1$ but $w(S)=0$ for every proper subset $S$ of $I$. Since $G(w)$ clearly equals $G$, w cannot be a valueable game so that BV is not a valueable space. From the above considerations we conclude that when looking for values on subspaces of nonatomic games one must either restrict the search to valueable subspaces, necessarily proper subspaces, or weaken the symmetric axiom or else combine these two approaches in an approprlate way. 
2. WEAKENING THE AXIOM OF SYMMETRY ON BV.

Let $H$ be a subgroup of $G$. An $H$-value on $B V$ is a linear positive efficient projection $\rho: B V \rightarrow F A$ which is H-symmetric. If an H-value exists then it follows that $H$ is a valueable group. For let $P$ be an $H$-value on $B V$ and let $w$ denote the unanimity game. Then we have

$$
H=H(w) \subset H(P w) \subset G(P w)
$$

This shows that $H$ is a measure group. We have just proved:

LEMMA 2.1. Every valueable group is a measure group.

In view of Lemma 2.1 it is natural to pose the following problem to which we have no answer.

PROBLEM 2.1. Is every measure group a valueable group? The first attempt to seek valueable groups was in Ruckle, [2] where it was shown that every locally finite group is a valueable group. The next two theorens strengthen that result.

THEOREM 2.1. Let $H$ be a subgroup of $G$. Then $H$ is a valueable group if and only if, the following condition is satisfied:

The group generated by every finite subset of $\mathrm{H}$ is a valueable group.

Next we place a topology on $G$ and define a subgroup $H$ of $G$ to be almost compact if every finitely generated subgroup of $H$ is compact in this topology. Using these notions we obtain the following result.

THEOREM 2.2. Every almost compact group is a valueable group.

If $H$ is a locally finite group then by Lemma $2.1, H$ is a measure group. This means there is $\mu$ in $F A^{\prime}$ such that $H \subset G(\mu)$. Sometimes it is desired that the value of a game be a countably additive measure and not just a member of FA. Thus the following question was posed by R.J. Aumann (Ruckle [2]): Is every locally finite group a measure group for some countable additive measure? This question is answered in Example 4 below where a locally finite group is constructed which does not belong to $G(\mu)$ for any $\mu$ in $\mathrm{CA}$.

\section{VALUES ON SUBSPACES OF BV.}

Most effort on values of nonatomic games has been dedicated to constructing values on subspaces of $B V$. The main existence and uniqueness results are the existence of a unique value on bv'NA (viz. Aumann and Shapley [1], the existence of a value on ASIMP (viz. Aumann and Shapley [1] weak ASIMP (viz. Neyman [3]) and the Mertens space (viz. Mertens [4]). Other than for a few results which are quite basic, there are no uniqueness theorems besides that for bv'NA. Thus the most pressing need in this area is for uniqueness results. Even the existence theory is far from satisfactory. The question of existence is in doubt for several "nice" spaces such as $\mathrm{AC}, \mathrm{pNA}^{\prime} \cap \mathrm{AC}$ and $\mathrm{AC}_{\infty}$ (viz. (Monderer [5]). We have already mentioned that a necessary condition for the existence of a value on $Q$ is that $Q$ be a valueable space. This leads to the following problem: 
PROLBEM 3.1. Does there exist a value on every valueable space?

Hoping for a positive answer to this problem we prove the following result:

THEOREM 3.1. The space $\mathrm{AC}$ is a valueable space. Moreover, for every $\mathrm{v}$ in $\mathrm{AC}$ we can find a $\mu$ in NA such that $\mu(I)=v(I),\|\mu\|<\|v\|, G(v) \quad G(\mu)$ and $\mu<<\lambda$ for every $\lambda$ in NA for which $v<<\lambda$. Moreover, if $v \in \mathrm{BV}^{+}$we can choose the corresponding $\mu$ to be in $\mathrm{NA}^{+}$.

\section{PROOFS OF RESULTS.}

We need the following Lemma.

LEMMA 4.1. Suppose $P$ is an efficient linear projection from BV onto FA. Then $\|P\|=1$ if and only if $P$ is a positive operator.

PROOF. Assume $P$ is a positive operator. For every $v$ in $B V$ we have

$$
\begin{aligned}
\left\|P\left(v^{+}\right)-P\left(v^{-}\right)\right\| & \leqslant\left\|P\left(v^{+}\right)\right\|+\left\|P\left(v^{-}\right)\right\| \\
& =\left(P\left(v^{+}\right)(I)+P\left(v^{-}\right)(I)\right.
\end{aligned}
$$

since $P\left(v^{+}\right)$and $P\left(v^{-}\right)$are in $\mathrm{FA}^{+}$by the positivity of $P$. Since $P$ is efficient we further note that

$$
P\left(v^{+}\right)(I)+P\left(v^{-}\right)(I)=v^{+}(I)+v^{-}(I)=\|v\|
$$

Therefore, we conclude that $\|\mathrm{P}\|<1$. As with all projections we know $\|\mathrm{P}\|>1$ (since $\|P\|=\left\|P^{2}\right\|<\|P\|^{2}$ ).

To establish the converse let $P$ denote an efficient projection with $\|P\|=1$. Suppose $P$ is a monotone game. Then $\mathrm{Pv}$ is equal to $\mu_{1}-\mu_{2}$ where $\mu_{1}=\left(\mathrm{Pv}^{+}\right)$and $\mu_{2}=(P v)^{-}$. We shall verify that $\mu_{2}(I)=0$ which implies $\mu_{2}=0$. Indeed, we have

$$
\mu_{1}(I)-\mu_{2}(I)=P V(I)=V(I)=\|v\|>\|P v\|=\mu_{1}(I)+\mu_{2}(I)
$$

from which we conclude that $\mu_{2}(I)<0$ so that $\mu_{2}(I)=0$.

The set of all efficient, positive, linear projections $P$ from BV onto FA will be denoted by $\Gamma$. For every subgroup $H$ of $G, \Gamma(H)$ denotes the set of all $P$ in $\Gamma$ which are H-values, i.e. $\pi^{-1}$ oPo $\pi=P$ for all $\pi \in H$. By Ruckle [2] $\Gamma$ and $\Gamma(H)$ are compact in the $w^{*}$-topology of operators in L(BV) - the space of all continuous linear operators from BV into itself. Moreover, $\Gamma$ is nonempty by Ruckle [2].

PROOF OF THEOREM 2.1. Let $H$ be a subgroup of $G$ which satisfies the condition state in the theorem. For every $\pi$ in $H$ let $\Gamma(\pi)$ be $\Gamma(D)$, where $D$ is the group generated by $\pi$. We shall prove that $\tau_{\varepsilon H} F(\pi)$ is nonempty. Since each $\Gamma(\pi)$ is compact, it suffices to prove that each finite intersection is nonempty. Indeed, if $E$ 
is the group generated by the finite subset $\left\{\pi_{1}, \pi_{2}, \ldots, \pi_{n}\right\}$ of $H$ we have $n_{i=1}^{n} \Gamma\left(\pi_{i}\right)=\Gamma(E)$, and $\Gamma(E) \neq \phi$ by the hypothesis of the theorem.

The converse of the theorem is obvious.

A subgroup $H$ of $G$ will be called a compact group if there is a topology $\tau(H)$ on $H$ for which (i) $\mathrm{H}$ is a compact topological group and (ii) the mapping from $\mathrm{H} X$ BV into BV defined by $(\pi, v) \rightarrow \pi(v)$ is continuous with respect to the product topology on H X BV and the Banach space topology on BV. Thus $H$ acts on BV as a group of continuous operators in the sense of Rudin [6]. For example, if $H$ is finite the discrete topology of $H$ satisifes these conditions. A subgroup $H$ of $G$ is called almost compact if every finite subset $\mathrm{F}$ of $\mathrm{H}$ is contained in some compact group $\mathrm{H}_{1}$.

PROOF OF THEOREM 2.2. Suppose $H$ is an almost compact subgroup of G. Every finitely generated subgroup of $H$ is contained in a compact group $H_{1}$. In order to apply Theorem 1.1. it suffices to prove that $H_{1}$ is valueable. This is a direct application of Theorem 5.18 of Rudin [6].

EXAMPLE 4.1. Let $I$ be the half open unit interval $[0,1)$. Let $I_{n}, n=1,2, \ldots$ be an infinite partition of $I$ into nonempty subintervals. For each $n>1$ let $H_{n}$ be the group of all symmetries $\pi$ of $I$ which satisfy the following conditions:

(1) $\pi$ is the identity on $U_{k=n+1}^{\infty} I_{k}$.

(2) $\pi$ is linear on each $I_{k}, k>1$.

(3) $\pi$ permutes the set $\left\{I_{1}, I_{2}, \ldots, I_{n}\right\}$.

Let $H$ be the union of $a 11 H_{n}, n>1$. Obviously $H$ is a locally finite group and if $H$ preserves some non-zero $\mu$ in FA, then $\mu\left(I_{j}\right)=\mu\left(I_{k}\right)$ for all $j$, $k$. Therefore $\mu$ cannot be in $\mathrm{CA}$.

One can prove that the group $H$ above preserves an FA' measure $\mu$ if and only if $\mu\left(I_{n}\right)=0$ for all $n$. For example, let $\lambda_{1}$ denote the normalized Lebesgue measure on $I_{i} \cdot$ Define $\mu$ by

$$
\mu(s)=\operatorname{LIM} n^{-1}\left\{\lambda_{1}(s)+\lambda_{2}(s)+\ldots+\lambda_{n}(s)\right\}
$$

where LIM is any Banach 1 imit.

PROOF OF THEOREM 3.1. Because of the standardness assumption we can, without loss of generality, assume $I$ is the unit interval $[0,1)$. For each $\mu$ in $N A$, let $N A(\mu)$ be the subspace of NA consisting of all measures which are absolutely continuous with respect to $\mu$. It is known that $\mathrm{NA}(\mu)$ is isometric to $L^{1}(\mu)$ via the isometry $\xi \rightarrow \frac{d \xi}{d \mu}$. Let $P: A C+N A$ be the operator for which we have $\operatorname{Pv}[0, s)=v[0, s)$ for every $s$. Thus $P$ is precisely the operator $\phi^{R}$ defined in (12.2) of Aumann and Shapley, [1] where $R$ is the natural order on $I$. For each $\pi$ in $G$ let $P^{\pi}$ be $\phi^{R}$, where $R$ is the order on $I$ defined by: $s<t$ if and only if $\pi^{-1}(s)<\pi^{-1}(t)$. Obviously we have, $P^{\pi}=\pi^{-1}$ oPo . $^{\circ}$ Let $v \varepsilon A C$; then by Proposition 12.8 of Aumann and Shapley [1], every $p \pi v$ satisfies 
the following conditions: $P^{\pi}(\mathrm{v})(\mathrm{I})=\mathrm{v}(\mathrm{I}),\left\|\mathrm{P}^{\pi}(\mathrm{v})\right\|<\|\mathrm{v}\|$ and $\mathrm{P}^{\pi}(\mathrm{v})<\mu$ for every $\mu$ such that $v \ll \mu$. Moreover, if $v$ is in NA then $P^{\pi}(v)=v$ and if $v \varepsilon \mathrm{AC}^{+}$ then $\mathrm{P}^{\pi}(\mathrm{v}) \in \mathrm{NA}^{+}$. Also it was proved in Aumann and Shapley [1] that for every $\mu$ in NA for which $v<<\mu$, the measures in $M v=\left\{P^{\pi} v: \pi \varepsilon G\right\}$ are uniformly absolutely continuous with respect to $\mu$.

Let $\mathrm{KV}$ be the closed (in the weak topology of $\mathrm{NA}$ ) convex hull of MV. Every $\nu$ in $\mathrm{Kv}$ satisfies the properties described above. Moreover, if $\mathrm{v}<<\mu$ then the members of $\mathrm{Kv}$ are uniformly absolutely continuous with respect to $\mu$. Therefore, by Theorem IV. 8.9 of Dunford and Schwartz [7], $\mathrm{Kv}$ is weakly compact in NA( $\mu$ ) for every such $\mu$. Now suppose that $v$ is in $A C$. Every $\pi$ in $G(v)$ maps $K v$ into itself since

$\pi P^{\tilde{\pi}}(v)=\mathrm{P}^{\tilde{\pi} 0 \pi^{-1}}(\mathrm{v})$. We now use the following fixed point theorem attributed to RyllNardzewski, see Glasner [8]. Let $K$ be a weakly compact convex subset of a Banach space $X$, and let $G$ be a group of continuous linear operators $\pi$ with $\pi(k)=K$ for each $\pi$ in $G$. If for every $x \neq y$ in $K$ we have

$$
\inf _{\pi \in G}|| n x-\pi y \|>0 \text {. }
$$

then there exists $\mathrm{x}$ in $\mathrm{K}$ such that $\pi \mathrm{x}=\mathrm{x}$ for all $\pi$ in $\mathrm{G}$.

Condition (2.1) is easily verified in the present case because each $\pi$ in $G$ is an isometry. Obviously every common fixed point of $G(v)$ is in $\mathrm{Kv}$ satisfies the conditions of the theorem.

\section{REFERENCES}

1. AUMANN, R.J. and SHAPLEY, L.S., Values of Non-Atomic Games, Princeton, Princeton University Press.

2. RUCKLE, W.H., Projections in certain spaces of set functions, Mathematics of Operations Research 7 (1982), 314-318.

3. NEYMANN, A., Weighted majority ganes have asymptotic values, Research Memorandum NR. 69, Center for Research in Mathematical Economics and Game Theory, The Hebrew University, Jerusalem.

4. MERTENS, J.F., The Shapley value in the non-differentiable case, Core Discussion Paper NR. 8240.

5. MONDERER, D., A milnor condition for nonatomic lipschitz games and its applications, working paper no. 113186. The Open University of Israel, Te1Aviv.

6. RUDIN, W., Functional Analysis, New York, McGraw-Hill, Inc.

7. DUNFORD, N. and SCHWARTZ, J.T., Linear Operators Part I, New York, Interscience 1959.

8. GLASNER, S., Proximal Flows, Berlin, Heidelburg, New York, Spinger Lecture Notes v. $517,1976$. 


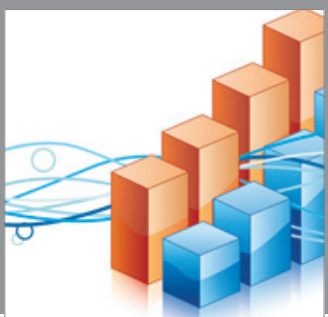

Advances in

Operations Research

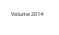

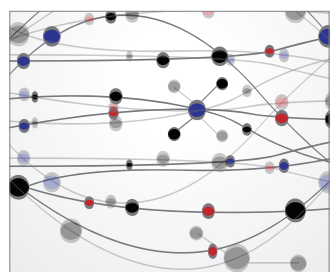

\section{The Scientific} World Journal
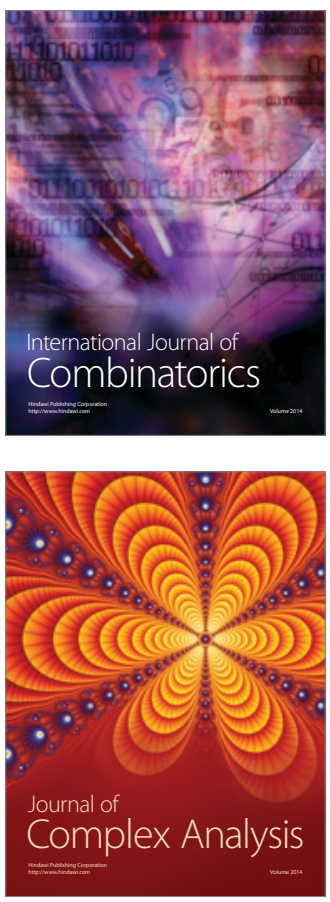

International Journal of

Mathematics and

Mathematical

Sciences
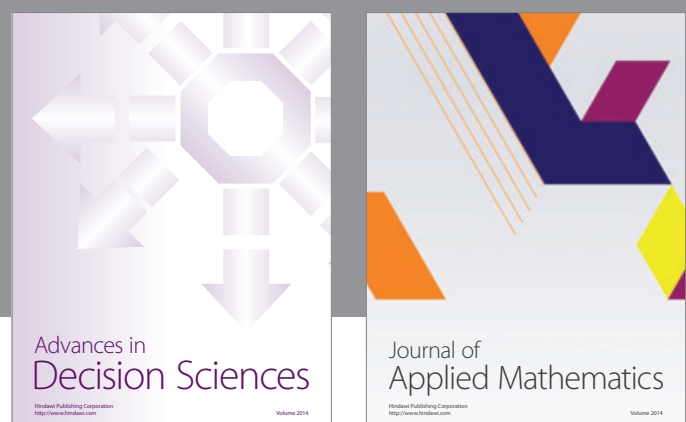

Journal of

Applied Mathematics
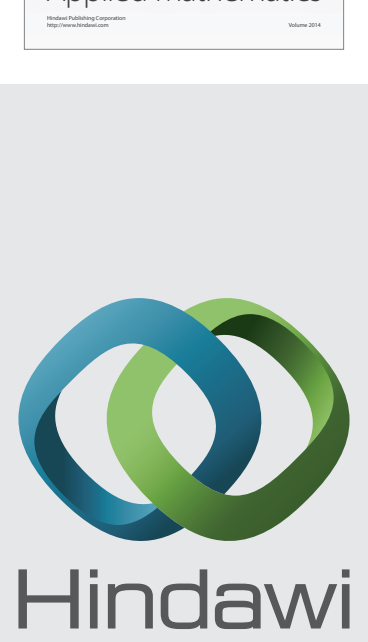

Submit your manuscripts at http://www.hindawi.com
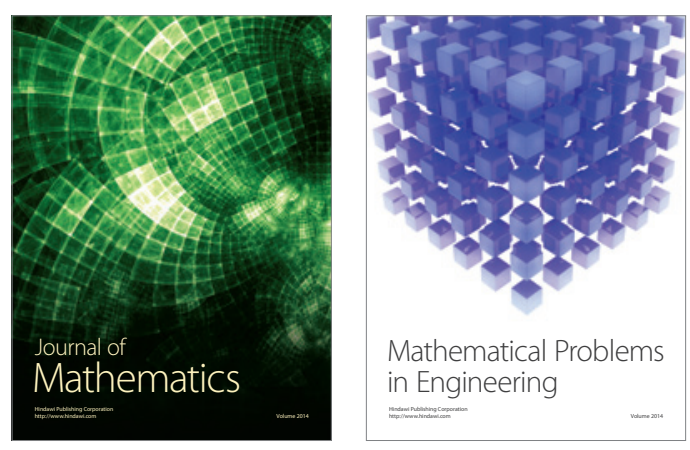

Mathematical Problems in Engineering
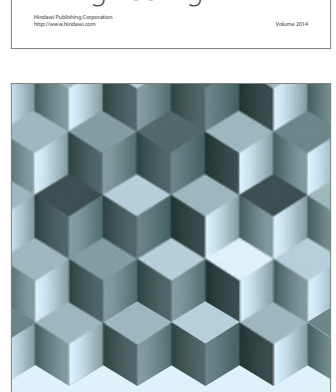

Journal of

Function Spaces
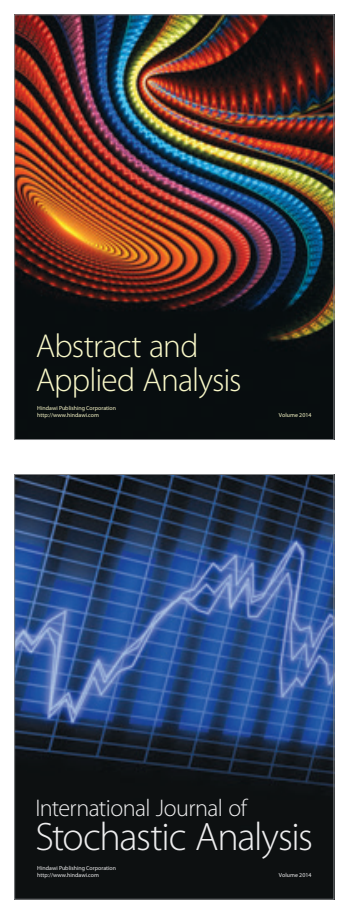

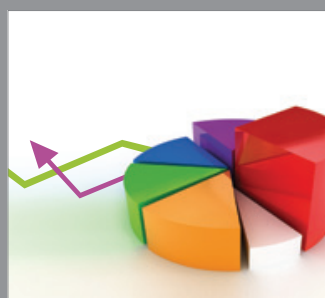

ournal of

Probability and Statistics

Promensencen
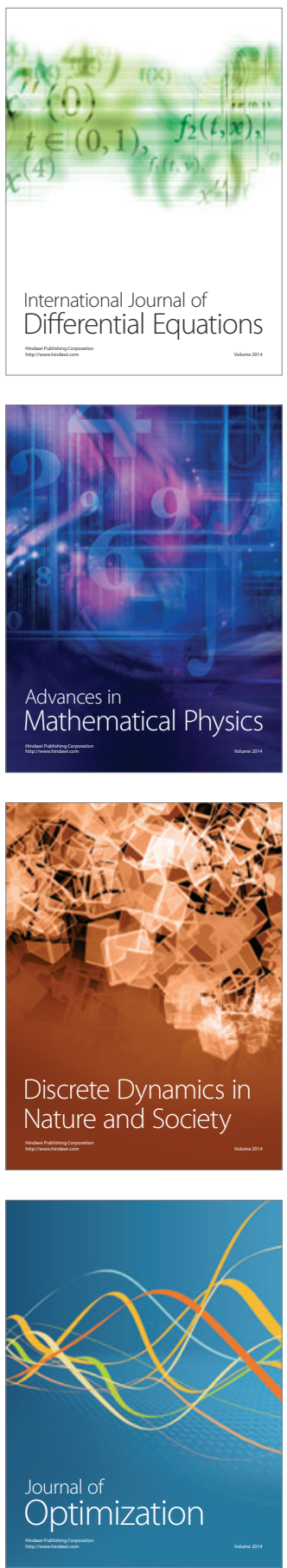